Instituto de la Vivienda. Información Estadística sobre la situación habitacioanl http://www.buenosaires.gob.ar/institutodevivienda erie-de-informes-situacion-habitacional-caba

\section{Legislación de la Ciudad de Buenos Aires}

Ley 148 sancionada en el año1998

Ley 324 sancionada en el año 1999

Ley 341 sancionada en el año 2000

Ley 1.251 sancionada en el año 2003

Ley 3756 aprobada en el año 2010

Ley 4219 aprobada en el año 2011

Ley 4.809 aprobada en el año 2013

Ley $N^{\circ} 5.724$ aprobada en el año 2016

Legislación Nacional

Ley Nacional № 17.174 sancionada en el año 1967

Cómo citar este artículo

Marazzi, Vanesa y Natalia Gualdoni (2017) “Las políticas públicas del hábitat popular en la Ciudad de Buenos Aires, 2011-2016”. Revista Perspectivas de Políticas Públicas vol. $6 \mathrm{~N}^{\circ}$ 12: 217-244

\title{
El Plan Estratégico de Modernización de la Administración Pública bonaerense. Un Análisis de los principios, los presupuestos y los modelos.
}

The Strategic Plan for the Modernization of Public Administration in Buenos Aires Province. Its fundamentals, assumptions and policy- patterns

Ricardo Sebastián Piana. Abogado y Doctor en Ciencis Jurídicas, UNLP.

Doctor en Ciencia P del Salvador. Docente (UNLP) Teoria y Derecho Constitucional (USAL) y del Sechinario de livestigación (UC r_piana@yahoo.es

Fecha de recepción:

10.1.17

Fecha de aceptación: 20.3.17

\section{Resumen}

El presente artículo analiza los principales puntos del Plan Estratégico de Modernización de la Administración Pública de la Provincia de Buenos Aire (Ley $\mathrm{N}^{\circ} 14.828$ ) que tiene por objeto instalar "un nuevo modelo de gestión de los recursos públicos". A diferencia de proyectos de anteriores gestiones, el actual gobierno de María Eugenia Vidal logró apoyo legislativo para instalar la perentoriedad de la reforma administrativa. El Plan se articula a través de un conjunto de programas, normas y procedimientos que cuestionan los modelos de funcionamiento burocrático de la Administración Pública bonaerense. A través del estudio de la norma se advierten los supuestos teóricos que sustentan estos cambios y el modelo de Estado buscado.

Palabras clave: Provincia de Buenos Aires - modernización administrativa - planeamiento administrativo - Ley 14.828 - estados subnacionales 


\section{Abstract}

This article approaches to the main aspects of the new Strategic Plan for the Modernization of Public Administration of the Province of Buenos Aires (Law 14.828), whose goal is to implement "a new model for the management of public resources". Unlike previous administrations, the incumbent one, headed by Governor María Eugenia Vidal gathered congressional support in order to promote it as an urgent matter. The Plan is articulated through a set of programs, norms and procedures that question the models of bureaucratic management in the Buenos Aires Province public administration. Through the study of the norm we will realize what the theoretical assumptions are that uphold these changes as well the pursued underlying scheme of state administration.

Key-words: Buenos Aires province - administrative modernization - administrative planning - Law 14.828 - subnational state administrations.

\section{Introducción}

Más allá de la reciente -y bienvenida- emergencia de estudios político-sociales a nivel subnacional, el análisis de las políticas de reforma en la Administración Pública en la provincia de Buenos Aires tiene un importante valor empírico, tanto por la representatividad de la unidad respecto del nivel nacional como por su preponderancia respecto de otras unidades provinciales.

No es necesario, en nuestro contexto, resaltar la importancia de la provincia de Buenos Aires en cuanto a su población, producción y superficie. Sí, tal vez, su carácter representativo de las propias diferencias que existen en todo el país: geográficamente ocupa menos del 10\% del territorio nacional, pero su población, que alcanza a 15.625.084 habitantes según el censo 2010, tiene el 38,9\% del total-país. Además, en ella puede verse reproducida la desigual distribución nacional de la población: en el conurbano, que ocupa una superficie de $3.630 \mathrm{Km}^{2}$, esto es, $1,2 \%$ de la superficie total, habita $63 \%$ de su población. Por ello, al igual que de la República Argentina, de la Provincia de Buenos Aires puede decirse que es un Estado macrocefálico.
En términos políticos, más allá del fenómeno de subrepresentación medida en cantidad de diputados nacionales que aporta al Congreso sobre cantidad de población (Reynoso, 2005), ${ }^{1}$ su número en términos absolutos no es nada despreciable: 35 diputados nacionales en cada elección. ${ }^{2}$

Por otro lado, esa magnitud poblacional hace que, como puede advertirse cada vez con más fuerza, las estrategias nacionales se "jueguen" en el territorio bonaerense. Su peso específico en el contexto nacional la hace estar presente en cualquier de los grandes acontecimientos nacionales. ${ }^{3}$ Sin embargo, su déficit presupuestario, ya crónico, la pone en una situación de inestabilidad institucional y dependencia política que debilitan esa magnitud que señaláramos.

La asunción de María Eugenia Vidal cerró un ciclo de hegemonía ininterrumpida del Partido Justicialista en la Provincia de Buenos Aires desde el año 1987 hasta la actualidad. Dos fenómenos aparentemente contradictorios caracterizan ese período: un marcado proceso de personalización del poder en torno a la figura del Gobernador, junto con una creciente territorialización de la política que le ha dado un fuerte poder a los intendentes, especialmente a los del Conurbano. Decíamos que la contradicción es aparente porque entendemos es la desinstitucionalización lo que ha derivado en una creciente "municipalización" de la política bonaerense. ${ }^{4}$

Otro de los ejes de este período ha sido la metamorfosis del Estado argentino ocurrido desde los años noventas (Orlansky, 1995, Oszlak, 2000; Cao y Esteso, 2001, Centrágnolo, 2004, Cao, 2008, entre otros). El gran cambio operó tanto en el Estado Nacional como en los provinciales modificando sus roles frente a la sociedad: si bien el traspaso de las responsabilidades por los servicios públicos, educativos, hospitalarios y de seguridad hacia las provincias puede resultar la nota más característica, otro tanto debe decirse respecto del perfil de su personal y la composición de su presupuesto, todo lo cual debió alterar sustancialmente su organización interna (Piana, 2012). Sin embargo, las reformas administrativas necesarias para soportar esta nueva estructura funcional no fueron de la mano.

Encerrada en la lógica de sobra-falta, la provincia de Buenos Aires no fue ajena a la gravedad de la crisis económica, política y social desatada en el país a partir de diciembre de 2001 ni a las corrientes teóricas de la Nueva Gestión Pública que propugnaron imponer las reformas administrativas en América Latina.

Precedido por un repaso por los antecedentes de proyectos que van justificando la necesidad de un tipo de reforma administrativa, analizaremos en las secciones que siguen el Plan Estratégico de Modernización de la Administración Pública de la Provincia de Buenos Aires y su Decreto Reglamentario recientemente aprobados, los supuestos fácticos y teóricos que sustentan estos cambios y cómo pretenden desmantelar los modelos 
de organización burocrática bonaerense planteando un conflicto de visiones sobre el funcionamiento del Estado provincial.

Para ello, precedido de un breve marco teórico, realizaremos un recorrido por los antecedentes provinciales en materia de modernización administrativa para adentrarnos en un análisis normativo de la reciente Ley de Modernización: sus fundamentos, presupuestos, principios y, finalmente, sus fuentes. Concluiremos que la norma propicia, en términos de política pública, un mimetismo con su norma similar de la Ciudad de Buenos Aires y adolece de identificación de problemas propios de la cultura administrativa bonaerense, optando por atender problemáticas generales de la forma burocrática. Sin embargo, el estudio de los antecedentes, nos permitirá concluir que desde la década de 1990 un mismo patrón teórico de reforma, el de la Nueva Gestión Pública, se ha intentado implantar en el ámbito de la Provincia de Buenos Aires.

\section{Aspectos teóricos en materia de reforma de la Administración}

Reforma, modernización y cambio aparecen generalmente expuestos como sinónimos. Pero como bien sostiene Groisman (1991:16),

... la reforma administrativa suele asociarse al concepto de modernización que es utilizado con cierta ligereza. Tiene una connotación positiva en cuanto está asociado la idea de progreso, de adaptación a los fenómenos contemporáneos, de recepción de nuevas técnicas, adelantos y descubrimientos. Pero entraña la posibilidad de convertirse en una noción ambigua o encubridora, de amparar la moda o de sobrevalorar la función técnica frente a la política.

De ahí la necesidad de estudiar las acciones que se han implementado en estos últimos años independientemente de su rótulo.

No existe una noción clara del concepto de modernización pese ser una de las temáticas claves de las ciencias sociales y jurídicas a diferencia del concepto modernidad sobre el que existe una abundante (aunque no siempre coincidente) bibliografia.

Conceptualizaciones tan amplias como las que aparecen en la voz Modernización del Diccionario de Política dirigido por Bobbio, Matteucci y Pasquino ${ }^{5}$ hasta los estudios de los trastornos sociales que producirían los procesos de anomia generados por los problemas de adaptación a las condiciones sociales industriales, ${ }^{6}$ pasando por los estudios de eficiencia y eficacia económica en matriz de producción económica, todos ellos hacen referencia a la modernización desde distintas ópticas y matices.
De forma amplia la modernización

...consiste en un cambio cada vez más acelerado e intenso de todas las estructuras sociales. Nuevas tecnologías, nuevos grupos -ascendentes y descendentes-, nuevos problemas, nuevas potencialidades, son el producto de la modernización. Como si se tratara del dibujo de una espiral, los parámetros de las situaciones posteriores son cambiados por los resultados de la actividad derivada de las situaciones anteriores

(Bouza-Brey, 1999:77) ?

Respecto al concepto de reforma, Julien Freund (1968) ha puesto de relieve la relación entre este concepto y el de revolución. La revolución adquiere un significado nuevo en la literatura política italiana de los siglos XIV y XV, en que la rivoltura o rivoluzione fue empleada como el primer equivalente de los trastornos negativos que la Edad Media llamaba seditio o rebellio, y más tarde, movimientos positivos de la reformatio, renovatio o restitutio. Y agrega:

Así, la literatura política italiana utiliza preferentemente el término [revolución] para designar la empresa que consiste en liberar una colectividad de un tirano para restablecer el antiguo orden. Los conceptos de reformatio, renovatio o restitutio contienen, asimismo, la idea de una vuelta a lo «verdaderamente antiguo» (Freund, 1968:720).

Como se advierte, asociado al concepto de reforma está tanto la idea de retorno como la de progreso. Sólo con la Ilustración y su fe constante en el poder de la razón humana se asociará la reforma sólo al progreso. En efecto, la idea de progreso es esencial para el pensamiento iluminista: supone la historia como un proceso en que los hombres alcanzan, lenta pero inexorablemente, una dirección definida y deseable y que ello será indefinidamente para gozar, en el término, de la felicidad. ${ }^{8}$

La gran mayoría de los autores hablan indistintamente de reforma del Estado y de la Administración Pública, sin embargo, mientras la primera observa, describe y analiza las funciones estatales en el contexto de los grandes cambios históricos-ideológicos que legitiman la relación de mando y obediencia, la segunda analiza las estructuras institucionales que ejecuta esas funciones; esto es, mientras la primera estudia qué hace el Estado, la segunda, cómo lo hace, o como lo ha dicho Hintze (2001:1) 
las decisiones sobre el qué hacer se refieren a las relaciones entre el Estado y la sociedad y las organizativas a cómo el aparato administrativo hace lo que hace".

Lo cierto es que muchas veces, en nuestros países, la reforma de la administración ha implicado una verdadera reforma del Estado: el achicamiento y aplastamiento de la pirámide; recorte de sueldos y jubilaciones; privatizaciones y transferencias al sector privado han implicado, modificaciones en el ámbito social, político y económico y por ello, no podemos dejar deslizar este error terminológico.

El ideario de la Nueva Gestión Pública se ha difundido a nivel mundial como eje central para el cambio y la modernización de las administraciones públicas en páses con tradiciones institucionales tan disímiles como Estados Unidos, Suecia, Gran Bretaña, Francia, Australia, incluyendo los de la propia región de América Latina aunque el resultado concreto de su aplicación ha adquirido escasa difusión (López, 2005). ${ }^{10}$

La Nueva Gestión Pública, como reacción a la Administración pública tradicional, ha buscado construir modelos de organización más flexibles a través de: 1) la desregulación, descentralización y delegación de funciones; 2) la desagregación de las áreas de competencia en agencias gubernamentales promoviendo su competencia y rivalidad y 3) la flexibilidad laboral (véase Heeks, 2001): un giro copernicano llevaría a cambiar al Estado como centro del sistema por el ciudadano, visto ahora como un cliente al que se le presta servicios. Se entiende que la legitimidad del Estado no estaría dada por su estructura institucional sino por su capacidad de prestar bienes y servicios. Desde esta lógica, sería necesario una administración con énfasis en los resultados y la satisfacción del ciudadano-cliente, lo que requería incorporar parámetros de medición de desempeño.

Se pretende que las burocracias obtengan mayor flexibilidad e inteligencia para actuar, sin perder el control de legalidad. Se sostiene que pasando del control de los recursos de los procesos a un nuevo criterio de control a través de evaluación de resultados, será posible planear el uso de los recursos y dirigir el gasto de esos recursos para obtener determinados resultados (Arellano Gault, 2001 y 2003).

Las consignas "post burocráticas" resultan sumamente atrayentes: su ejercicio conduciría a un gobierno más económico y eficiente, con servicios de mayor calidad y programa más eficaces, y además, simultáneamente, introduce cambios como la ampliación del control político, mayor libertad a los gerentes públicos para que lleven a cabo su gestión, mayor transparencia gubernamental, postulados éstos que nadie podría renegar. Además, los principios y técnicas que configuran el management público se presentaron como un conjunto de iniciativas aplicables a todo tipo de organizaciones y caracterizadas por su neutralidad política (Piana, 2012 b).
Pareciera que el acento puesto en el control es totalmente nuevo y propio de una visión gerencial del Estado, cuando en realidad es tan antiguo como la administración misma. Lo que sí es nuevo, es la

..tendencia a considerar el control de la gestión pública, sobre todo en la parte presupuestaria, como una suerte de competencia técnica pura, donde aplicando ciertas metodologías de control, se solucionarían cuestiones claves como son, entre otras, la distribución de los recursos, las responsabilidades de ejecución y el logro de las metas planteadas en un cierto período de tiempo establecido (Cleary, 2006:2).

Se señala que la Nueva Gestión Pública es una colección de ideas valores y creencias prestados de diferentes escuelas del pensamiento y de diferentes corrientes administrativas (Vargas Hernández, 1999).

Varias corrientes integran la Nueva Gestión Pública: tal vez la más conocida de ellas sea el gerencialismo cuya impronta está dada por la incorporación en el ámbito público de la lógica del mundo empresario y gerencial. Uno de los principios sobre el que se ha trabajado con mayor insistencia desde esta visión es dejar que los gestores gestionen buscando incrementar la responsabilidad de los cargos técnicos, por un lado, delegando más poder de decisión basado en la satisfacción del cliente, por el otro. En definitiva, la Nueva Gestión Pública busca romper con las estructuras compactas y piramidales de la administración por unidades independientes y autogestionables. Se pretende que la competencia propia del sector privado se dé entre dichas agencias del sector público, competencia que sería impulsada mediante premios y castigos en las asignaciones de recursos conforme a los niveles de eficiencia alcanzados. ${ }^{11}$

Debemos destacar que los principios y técnicas que configuran el "management público" se presentaron como un conjunto de iniciativas aplicables a todo tipo de organizaciones y caracterizadas por su "neutralidad política". Sin embargo, la supuesta neutralización de las administraciones sobre un modelo de corte científico y de racionalidad técnica que el gerencialismo propicia, es un supuesto teórico dificilmente contrastable. Las prácticas reales demuestran que las administraciones tienen un fuerte elemento de cultura política y organizacional, el cual es necesario apreciar a fin de institucionalizar cualquier proceso de reforma, por un lado, y que estos proyectos de reforma tienen un alto componente político. ${ }^{12}$

Es en este período y contexto que iniciamos el estudio de los antecedentes de la actual Plan Estratégico de Modernización de la Administración Pública pues, como veremos, 
tiene muchos aspectos en común con las propuestas de reformas administrativas previamente propiciadas.

\section{Los antecedentes de reformas en la Provincia de Buenos Aires}

Durante el período 1990-1999 se implementaron diversas reformas en el Gobierno provincial que siguieron los patrones de la reforma del Estado "de primera generación". ${ }^{13}$

Con la Ley N 11.184 de Reconversión Administrativa, sancionada en 1991, la Provincia de Buenos Aires se dio su propia ley de reforma del Estado, siguiendo el modelo del Estado nacional, aunque con dimensiones mucho más acotadas y con menor jerarquía en la agenda pública. ${ }^{14}$

Dicha ley autorizó al Poder Ejecutivo a la intervención de entes o empresas del Estado provincial para racionalizar sus estructuras administrativas; optimizar recursos y servicios; disponer su transferencia al sector privado y fijar una política de compromiso con el Poder Ejecutivo a través de "contratos programa". Por el artículo $8^{\circ}$ se facultó al Poder Ejecutivo a crear entes autárquicos institucionales que absorbieran, total o parcialmente, competencias de órganos de la Administración Central, con lo que identificaba a la Administración centralizada como un disvalor. Se lo autorizó, asimismo, a reubicar cargos de igual o superior jerarquía y agrupamiento al personal comprendido o reasignar su destino. ${ }^{15}$

Fue también en el marco de dichas políticas que el Gobierno Nacional en 1993 transfirió a la Provincia el manejo de las redes ferroviarias de transporte de pasajeros para su posterior privatización. Un año después se trasfirió Astilleros Río Santiago, pero la privatización no pudo concretarse. Este proceso se continuó en años posteriores: en 1997 privatización de la "Empresa Social de Energía Eléctrica de Buenos Aires" (E.S.E.B.A.) y privatización de "Obras Sanitarias de la Provincia de Buenos Aires" (O.S.B.A.) en 1999. Como consecuencia de ello, se crearon organismos reguladores y de control. Se inició también un proceso de transferencia de la ejecución del llamado gasto social, que comprendió desde los establecimientos de salud y educación hasta la gestión de algunos fondos nacionales (Fondo Nacional de la Vivienda, Fondo Especial de Desarrollo Eléctrico del Interior, entre otros)

En el ámbito del propio Poder Ejecutivo, el decreto $N^{\circ}$ 18/91 estableció nuevos criterios para el diseño y aprobación de estructuras organizativas y creó el Consejo Asesor de la Reforma del Estado y Procedimientos Administrativos (CAREPA). En los fundamentos de la norma se establecieron como objetivos mejorar el perfil operativo de la Administración Pública Provincial, desburocratizar el funcionamiento de los Departamentos de Estado, imprimir celeridad al trámite de reorganización de los mismos en el marco del "inicio de una nueva etapa de la reforma del Estado Provincial que busca dar a la misma el máximo de eficiencia y actuar con la idoneidad que ésta requiere" (fundamentos del decreto)

Luego de la crisis de 2001, el impulso de la reforma volvió a cobrar centralidad, con similares diagnósticos y objetivos. Durante la primera Ley de Ministerios de la gestión del Ing. Felipe Solá como gobernador de la provincia se creó una Secretaría para la Modernización del Estado que ocupó el Contador Florencio Randazzo, quien llegó a ocupar la Secretaría de Gobernación luego de haberse desempeñado en la jefatura de Gabinete de Ministros de la provincia.

El "Plan Rector de Modernización" elaborado por el Poder Ejecutivo a través de esta Secretaría cuando estaba a cargo de Randazzo, fue presentado en 2003. El Plan se presentó como un anteproyecto de ley, pues muchas de las reformas requerían modificación de las leyes vigentes.

El proyecto estaba compuesto por un conjunto de programas, normas y procedimientos

destinados a instalar una administración de los recursos públicos orientada a los resultados y a la responsabilidad de los funcionarios frente a los mismos. Representa un nuevo modelo de gestión que se sustenta en la panificación y el control, la reforma de los sistemas administrativos, y en la transparencia, la participación y el control ciudadano en la gestión pública

(Randazzo, 2004:58).

El Plan estaba estructurado en dos programas centrales con sus respectivos componentes; el primero de ellos asociado a la modernización Administrativa y el segundo a la Transparencia y Participación Ciudadana:

- Programa de Modernización Administrativa: Se integraba por un conjunto de herramientas y técnicas de gestión de recursos humanos, financieros, fisicos y tecnológicos destinados a fortalecer la capacidad administrativa y la eficacia institucional de la Administración Pública Provincial. El objetivo manifiesto era implementar una "gestión por resultados" y así lograr: mayor eficacia, eficiencia, economía y calidad en el cumplimiento de políticas, objetivos y metas; ordenamiento y control en el uso y disposición de los recursos públicos asignados. ${ }^{16}$

- Programa de Transparencia y de Participación Ciudadana: Incluía un conjunto de herramientas destinadas a involucrar a los ciudadanos y las organizaciones de la sociedad 
civil en la formulación, la ejecución y el control de la Administración Pública introduciendo normas y sistemas de transparencia. ${ }^{17}$

El Plan Rector introducía el concepto de control interno dentro de una visión gerencial del funcionario. Según el Plan, existirían "gerentes públicos" en quienes recaerían la responsabilidad de administrar recursos públicos y garantizar resultados de gestión, tanto en términos de bienes y servicios brindados como en términos del impacto producido por éstos en la comunidad, debiendo responder por la responsabilidad asignada y por los resultados de la gestión.

Dentro del Plan, se diseñaron Acuerdos Programa como herramientas mediante las cuales los organismos se comprometerían a alcanzar determinados resultados de gestión, teniendo como contrapartida el otorgamiento de una serie de flexibilidades en materia presupuestaria. Se preveía que los Acuerdos Programa fueran suscriptos por los titulares de organismos y de unidades ejecutoras de programas presupuestarios bajo la supervisión del Ministerio de Economía. ${ }^{18}$ Estas especies de "contratos" hacia dentro de la Administración, la gestión por resultados así como la figura de los gerentes públicos, son un claro reflejo de las políticas de la Nueva Gestión Pública, más específicamente, de una de sus corrientes, el gerencialismo, que se pretendió imponer en Latinoamérica en esa misma época.

Pese al fuerte "posicionamiento" político del Plan de Modernización y de la presentación del Proyecto, el plan no tuvo acogida legislativa. Según lo reconocía Randazzo (2004), se habían presentado muchas dificultades políticas. Si bien hasta ese momento el sistema político bonaerense podía calificarse por una oposición legislativa débil a los proyectos del Ejecutivo, ${ }^{19}$ en su primer período el gobernador Solá

"carecía entonces de poder político propio y dependía de la fuerza del propio presidente Duhalde, quien controlaba al Partido Justicialista en esa provincia"

(Godio, 2003:79).

Solá no gozó de los beneficios de la hegemonía del partido, pues pese a contar con mayoría formal en ambas Cámaras, la disciplina partidaria se encontró en crisis como consecuencia de la ruptura que se produjo entre Eduardo Duhalde y Néstor Kirchner. ${ }^{20}$

Distinto ha sido el proceso de llegada al ejecutivo bonaerense de Daniel Scioli y el contexto en el cual gobernó. Pese al ostracismo al que había sido conminado en su cargo de Vicepresidente de la Nación durante el gobierno de Néstor Kirchner, supo recomponer esa relación y ser nominado como candidato a Gobernador, contando con fuerte apoyo popular y en las urnas ${ }^{21}$ aunque con un relativo poder territorial. La fórmula Scioli-
Balestrini se impuso con el apoyo del 48,24 \% de los votantes y renovó su cargo en 2011, con Gabriel Mariotto como compañero de fórmula, con el 55,06\% de los votos con un amplio margen respecto a la segunda fórmula más votada. ${ }^{22}$

En materia de reforma del Estado y de la administración, antes de asumir contó con el apoyo de las Cámaras para aprobar una nueva Ley de Ministerios ( promulgada antes de la asunción, el 27/11/07) la ley de Administración Financiera (ley 13.767, promulgada el día de su asunción), y dos años después una nueva Ley de Contrataciones ${ }^{23}$, sustituyendo estas dos últimas a la vetusta pero tradicional Ley de Contabilidad de la Provincia del año $1971 .{ }^{2}$

Anunciado públicamente en 2010, la entonces gestión provincial presentó en marzo 2011 un proyecto de ley Marco para la Regionalización de la Provincia de Buenos Aires, cuyo objetivo era

impulsar su desarrollo institucional, económico y social mediante el despliegue de las capacidades de cada una de las regiones, para disminuir desequilibrios en materia de desarrollo socioeconómico, crecimiento poblacional e infraestructura, mejorando el tejido social con políticas públicas adaptadas regionalmente y para lograr un mayor acercamiento del Estado Provincial a sus habitantes, a través de un proceso de transformación del Estado Provincial profundo y abarcativo

(art. $2^{\circ}$ del proyecto de Ley Marco de Regionalización)..$^{2 .}$

Entre los fundamentos del proyecto se señala la necesidad de realizar una profunda reforma institucional. Se añade que el proyecto supone un nuevo paradigma de Estado y una transformación del Estado provincial. Allí se indica que la concentración de responsabilidades en la administración central resta eficacia a su accionar ya que las funciones de planificación y control han ido cediendo espacio a la resolución de innumerables cuestiones de tipo operativo, que bien podrían ser delegadas en niveles ejecutivos inferiores. Otro de los fundamentos, críticos del actual funcionamiento del Estado bonaerense, es la lentitud en los procedimientos mientras "La ciudadanía demanda soluciones, pide resultados, quiere un Estado presente, que defina estrategias y sea a la vez ágil, inteligente, moderno, eficaz, que rinda cuentas" (Fundamentos del proyecto de Ley Marco de Regionalización).

En cuanto a contenido, el proyecto es muy abierto delegando muchas de las acciones a la reglamentación del Ejecutivo, a diferencia del proyecto anteriormente analizado que contaba con más de 120 artículos. 
En los fundamentos aparecen distintos conceptos: transferencia de funciones, articulación y mediación, desconcentración y descentralización, competencia territorial y autarquía. ${ }^{26}$ En cada una de estas regiones, a cargo de ejecutivos regionales, aparecen competencias en temas relacionados con educación, salud, infraestructura, medio ambiente, deporte, turismo, obra pública, producción, agricultura y ganadería, entre otros, absorbidas de aquellas con las que actualmente cuentan los Ministerios. ${ }^{27}$ Pero en el esquema propuesto, se adopta una estructura matricial: las regiones dependerán jerárquicamente del Ministro Coordinador de las Regiones pero funcionalmente de los Ministerios centrales que desconcentren o deleguen en ellas funciones a través de Acuerdos o Convenios de Gestión (AG) que incluyan compromisos e incentivos sobre el esquema de gestión basada en resultados. Es decir, las funciones de los ejecutivos regionales dependen de la delegación mediante acuerdos entre los Ministerios centrales y el Ministro Coordinador de las Regiones (Piana, 2015).

La adscripción ideológica del modelo estatal a la nueva gestión pública es explícita: cuando propone la adopción de sistemas de gestión

orientados a resultados, estructuras matriciales, relaciones funcionales y jerárquicas, planeamiento estratégico, etc., son medios $\gamma / 0$ herramientas que permiten potenciar la gestión del Estado en el territorio y de las unidades regionales con el Estado Central"

(Buenos Aires,Plan de Regionalización ...,2011:31).

Más adelante volvemos a encontrar esta fundamentación y adscripción ideológica:

.. el actual esquema propone reemplazar el modelo tradicional de organización y entrega de servicios públicos, basado en los principios de la jerarquía burocrática, la centralización y el control directo, por una nueva gerencia pública que fortalezca la eficiencia y eficacia. En otras palabras, promueve ir de la administración pública a la gerencia pública

(Buenos Aires, Plan de Regionalización ..., 2011:40).

Así, tanto desde las líneas argumentales como desde los instrumentos, el Plan de Regionalización se adscribe claramente a las tendencias de la Nueva Gestión Pública.

El proyecto de ley fue aprobado en la Cámara de Diputados de la Provincia en noviembre de 2011 pero, girado en 2012 y pese a ser especialmente nombrado en el Discurso de apertura de sesiones de ese año por el Gobernador Scioli, no se aprobó en el Senado.
Una serie de audiencias demoró la reforma esperada por el Ejecutivo y el trámite perdió estado legislativo.

\section{El Plan Estratégico de Modernización de la Administración Pública}

La gobernadora que asumió en diciembre 2015 sostuvo en su discurso de apertura del año legislativo, el $1^{\circ}$ de marzo de 2016 , que ...

Algunos de ustedes tienen muchos años en la política y saben que la Provincia de Buenos Aires hace décadas que tiene problemas estructurales. Económicos, de falta de inversión, con un Estado obsoleto en términos administrativos y con una deuda social enorme. [...]

Encontramos un Estado que está más preparado para obstaculizar que para gobernar, con un sistema de administración obsoleto, burocrático y lento. [...]

Muchos de los proyectos más importantes van a pasar por este recinto legislativo. Vamos a proponer una Ley de Modernización del Estado, que nos va a permitir incorporar tecnologías de información y comunicación para tener una gestión activa $y$ transparente. Y eso se va a traducir en mejores servicios, por ejemplo, una ventanilla única para la gestión de trámites de todos los ciudadanos. ${ }^{28}$

Antes de iniciar con el análisis del Plan, debemos destacar dos antecedentes. El primero de ellos, a primera vista muy menor, es la sanción del Decreto $\mathrm{N}^{\circ} 37 / 15 \mathrm{~B}$ que deroga el Decreto $N^{\circ} 1322 / 05$ que regulaba el régimen para la formulación, financiación, presentación, tramitación y aprobación de estructuras de la administración pública provincial. Si bien el conocido como "Decreto de estructuras" era uno de los decretos más excepcionados, ${ }^{29}$ marcaba un hito del buen modelo de organización de las estructuras administrativas a partir del cual las desviaciones debían ser justificadas y no dejaban de advertirse. Ese Decreto no fue reemplazado por otro esquema lo que permite una mayor flexibilidad en el diseño de las actuales estructuras.

El segundo antecedente es la aprobación a mediados de abril de 2016 de la Ley de Emergencia Administrativa y Tecnológica (ley $\left.\mathrm{N}^{\circ} 14.815\right),{ }^{30}$ que modifica sustancialmente los procesos de compra y la contratación de personal bajo el régimen de servicio en los términos de la emergencia declarada por su artículo $1^{\circ}$. En ese mismo artículo se señala que la ley tiene por finalidad paliar el grave déficit en materia administrativa y tecnológica 
que en el transcurso de los últimos años se ha visto incrementado progresivamente y posibilitar la realización de las acciones tendientes al fortalecimiento y modernización tecnológica.

El proyecto de la que fuera posteriormente sancionada como Ley de Emergencia, elaborado por el Ejecutivo, entre sus fundamentos señala que la Provincia

se encuentra en una crítica situación que afecta a la totalidad de las áreas administrativas dependientes de Poder Ejecutivo Y, especialmente, a la prestación de los servicios esenciales a cargo de la Administración Pública Provincial, centralizada y descentralizada; extremos que son de público conocimiento y que responden a años de desinversión y de administraciones imperfectas, provocando una actuación estatal deficiente e ineficaz.

Como señaláramos, esta Ley introdujo la posibilidad de contratación de personal bajo la modalidad de locación de servicio regulado exclusivamente por las pautas del contrato sin vinculación con la ley de empleo público provincial, Ley № 10.430. También modificó esta última alterando las pautas salariales del personal de gabinete, el que podría tener libertad salarial entre sí y hasta un monto inferior al funcionario al que asiste. Esta ley, junto con el Decreto $N^{\circ} 230 / 16^{31}$ de delegación de funciones a los Ministros, entre otros, limitando las comisiones de servicio, y el Decreto $\mathrm{N}^{\circ} 618 / 16^{32}$ de congelamiento de la planta permanente y de la planta transitoria por un año, prorrogable por otro año más, generaron fuerte rechazo sindical.

El logro de una eficiente y eficaz descentralización administrativa, sanear el déficit fiscal y normalizar la gestión de las distintas prestaciones y servicios estatales para cumplimentar los objetivos de gobierno fueron algunas de las fundamentaciones de estas normas.

No muy distintos son los fundamentos de la ley que aprueba el "Plan Estratégico de Modernización de la Administración Pública de la Provincia de Buenos Aires" que fuera sancionada a inicios de julio de este año y registrado bajo el número de Ley $\mathrm{N}^{\circ}$ 14.828..$^{33} \mathrm{El}$ proyecto obtuvo un alto consenso en la Legislatura: fue aprobado por unanimidad en ambas Cámaras. La única modificación relevante introducida en el Legislativo al proyecto elevado por el Ejecutivo fue el referente a que la aplicación del Plan Estratégico de Modernización no implicará el despido de trabajadores $\gamma / 0$ trabajadoras. Dejándose a salvo las disposiciones previstas en la Ley $\mathrm{N}^{\circ}$ 10.430 y modificatorias, las que será de aplicación plena" (art. 7 , in fine, Ley $\mathrm{N}^{\circ} 14.828$, SIC).

Liminarmente entre los fundamentos se señala que el

proyecto responde a la imperiosa necesidad de adecuar los procesos administrativos a los sistemas de calidad, herramientas informáticas y buenas prácticas de gestión administrativa actualmente vigentes a nivel regional y mundial".

Se afirma que modernizar la Provincia conllevará desplegar servicios innovadores en el sistema de salud, seguridad, escuelas y producción. Se afirma la necesidad de

brindar servicios al ciudadano de forma rápida y eficiente, priorizando tiempos, evitando la superposición de trámites y agilizando procesos.

Señala el primer artículo de la Ley que el "Plan Estratégico de Modernización del estado" (SIC) estará compuesto por un conjunto de programas, normas y procedimientos destinados a instalar, en el ámbito de la provincia de Buenos Aires, un nuevo modelo de gestión de los recursos públicos sustentado en la planificación y control, en el proceso de reforma de los sistemas administrativos, a fin de lograr una administración orientada a los resultados, y a la transparencia y control ciudadano en la gestión pública. Según la ley los principios rectores que orientan el "Plan Estratégico de Modernización de la Administración Pública de la Provincia de Buenos Aires" son cinco: calidad, optimización en el uso de los recursos públicos, economía y racionalidad administrativas, ética y transparencia y participación de la ciudadanía en la gestión pública para el fortalecimiento de la democracia ${ }^{34}$

La propia ley además de fijar en el Ministerio de Coordinación y Gestión Pública la aplicación del Plan y asignarle funciones, establece que deberá propiciar un "esquema de desconcentración administrativa y descentralización territorial del gobierno provincial" además de elaborar un plan de capacitación, formación y perfeccionamiento continuo para todos los agentes de la Administración Pública Provincial.

En cuanto al propio objeto del Plan, aprobado como Anexo de la Ley, se señala

Alcanzar una gestión pública de calidad que posibilite la provisión eficaz y eficiente de bienes y servicios pú- 
blicos a los ciudadanos de la provincia de Buenos Aires de manera equitativa, transparente y efectiva, para una mayor integración y desarrollo de la sociedad, impulsando la ejecución de sistemas de conducción sistemáticos $y$ coordinados y el uso intensivo de las Tecnologías de la Información y las Comunicaciones (TICs) por parte del Estado Provincial

(art. $1^{\circ}$ Anexo).

Casi a renglón seguido se delinean los 5 ejes de la Reingeniería del Estado ${ }^{35}$ Provincial que por su relevancia, transcribimos in extenso:

Anexo. Artículo $3^{\circ}$.- Ejes de la Reingeniería del Estado Provincial:

Los ejes serán los siguientes:

a) Transformación institucional: Implementación de políticas públicas con miras al mejoramiento de la calidad de vida y al desarrollo integral de los ciudadanos de la provincia de Buenos Aires, promoviendo e introduciendo el uso de las nuevas Tecnologías de Información y Comunicación (TICs), con el objeto de fomentar la colaboración y coordinación en el ámbito de la administración pública, de manera de responder con mayor celeridad $y$ efectividad a las demandas de la sociedad.

b) Participación Ciudadana: es el marco para la relación entre los organismos públicos y los ciudadanos, con la finalidad de permitir a los ciudadanos realizar consultas, solicitudes, sugerencias y reclamos ante el Gobierno de la provincia de Buenos Aires a través de un sistema accesible vía internet, telefónica y mediante cualquier otro medio que se considere oportuno.

c) Mejorar la gestión pública: vinculación entre la formulación de planes y acciones y la programación presupuestaria, articulando la toma de decisiones cotidiana, el planeamiento estratégico, el proceso de programación presupuestaria, la reingeniería de procesos y sistemas de información de cada organismo y la promoción de la arti- culación entre éstos, el monitoreo de gestión y la rendición de cuentas por resultados, para lograr una administración pública eficiente y transparente.

d) Orientar el servicio de la función estatal en todos los aspectos de su actividad al servicio de los ciudadanos: a través de la implementación de canales efectivos de participación y control, mediante la utilización de las TICs para simplifcar y facilitar la vinculación de los ciudadanos con el Estado Provincial, mejorar la calidad de dicha relación y reducir los tiempos y costos involucrados en las transacciones.

e) Impulsar la implementación de la Modernización de la tramitación administrativa mediante las siguientes acciones: I) el desarrollo de nuevos sistemas de gestión; II) modernización de los procedimientos de compras, contrataciones $\gamma$ distribución de bienes $\gamma$ servicios; III) ejecución del proceso de transformación documental en miras a la gestión documental electrónica con procesos de innovación con impacto directo sobre la sustentabilidad de los procesos y los recursos, propendiendo a la progresiva despapelización en beneficio del ciudadano; IV) la simplificación de trámites, reducción de tiempos de tramitación y gestión y V) puesta en funcionamiento de una ventanilla única que agilice las posibilidades de acceso de los ciudadanos a la información.

Estos ejes deben cruzarse con los tres componentes que se numeran en el artículo $4^{\circ}$ del Plan y que conforman su núcleo duro: a) sistema de gestión por resultados; b) atención ciudadana y participación; c) Gobierno electrónico y nuevas tecnologías de la información y comunicación. ${ }^{36}$ Estos componentes son, en verdad, las actividades concretas o "instrumentos de gestión" como neutralmente los identifica el Plan y aparecen en Capítulos del Título II del Plan con descripción, en cada caso del instrumento, el objetivo y las actividades.

Si comparamos la Ley bonaerense con la ya aprobada en la Ciudad de Buenos Aires (ley No 3304 de 2009), advertimos muchas similitudes. Los objetivos generales de ambos cuerpos legales son similares como también a los componentes, con excepción del referido a la Gestión Integral de los Recursos Humanos, que no encontramos en la Provincia y que no hubiera sido acogido, a la luz del agregado hecho en la Cámara Legislativa y las protestas sociales respecto a la Ley de Emergencia Tecnológica y Administrativa. ${ }^{37}$ 
Los objetivos de las componentes y las acciones también coinciden. La única gran innovación son los Ejes de la Reingeniería, que hemos transcripto más arriba.

El siguiente cuadro resume los instrumentos de gestión, los objetivos y las actividades de cada componente de la Ley, habiendo resaltado los elementos originales de la bonaerense.

Cuadro 1. Componentes-Instrumentos de Gestión y Actividades del Plan Estratégico de Modernización de la Administración Pública- Ley $\mathbf{N}^{\circ} 14.828$

\begin{tabular}{|c|c|c|c|}
\hline Componentes & $\begin{array}{l}\text { Instrumentos de } \\
\text { gestion }\end{array}$ & Objetivo(s) & Actividad(es) $)^{38}$ \\
\hline \multirow{4}{*}{$\begin{array}{l}\text { Sistema de } \\
\text { gestion por } \\
\text { resultados }\end{array}$} & $\begin{array}{l}\text { Planes Estratégicos } \\
\text { de Gestion }\end{array}$ & 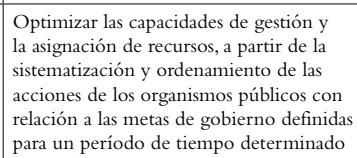 & 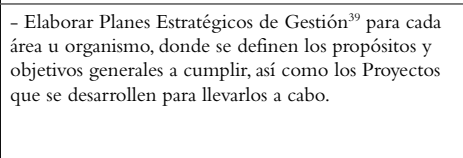 \\
\hline & $\begin{array}{l}\text { Planes } \\
\text { Operativos } \\
\text { Anuales }\end{array}$ & 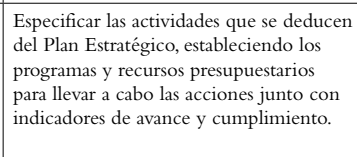 & $\begin{array}{l}\text { - Elaborar un Plan Operativo Anual con objetivos y } \\
\text { metas espećificas anualmente, junto con indicadores de } \\
\text { avance, de cumplimiento y presupuestarios. } \\
\text { - Elaborar Compromisos de Desempeno entre } \\
\text { los responsables smáximos de los organisismos o } \\
\text { dependencias }\end{array}$ \\
\hline & $\begin{array}{l}\text { Reingeniería de } \\
\text { procesos operativos, } \\
\text { administrativos } \\
\text { y de control. }\end{array}$ & 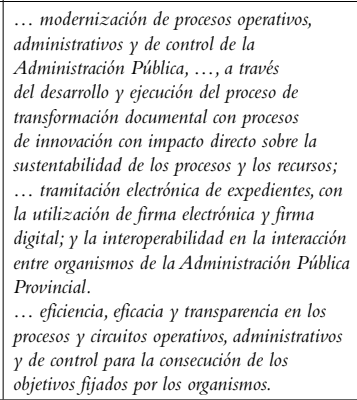 & 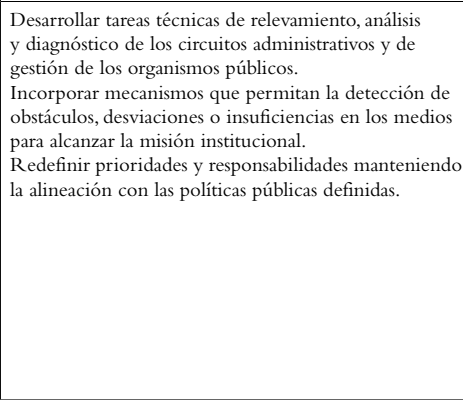 \\
\hline & $\begin{array}{l}\text { Tableros } \\
\text { de Control }\end{array}$ & 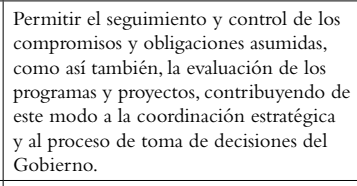 & 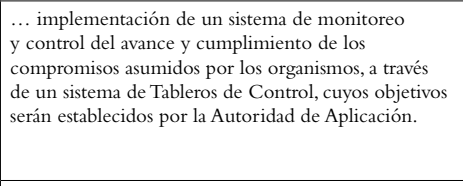 \\
\hline $\begin{array}{l}\text { Simplification de } \\
\text { laAdministración } \\
\text { Püblica }\end{array}$ & & 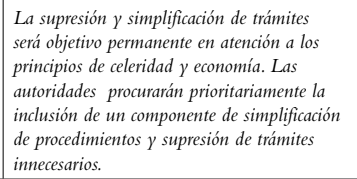 & \\
\hline
\end{tabular}

\begin{tabular}{|c|c|c|c|}
\hline & $\begin{array}{l}\text { Sistema de Consultas, } \\
\text { solictiddes, sugerencias } \\
y \text { rectlamos }\end{array}$ & 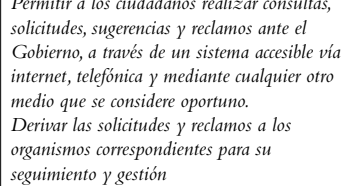 & 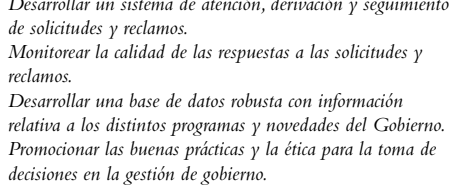 \\
\hline & $\begin{array}{l}\text { Ventanilla Única } \\
\text { de Trámites }\end{array}$ & 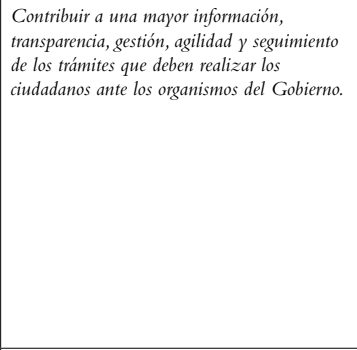 & 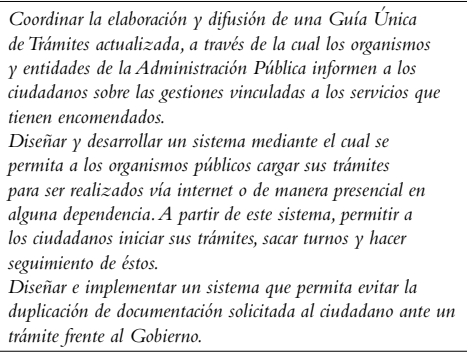 \\
\hline $\begin{array}{l}\text { Mecanismos } \\
\text { de atenciön }\end{array}$ & $\begin{array}{l}\text { Programa de Calitad } \\
\text { en la Gestión }\end{array}$ & 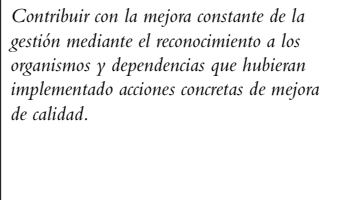 & 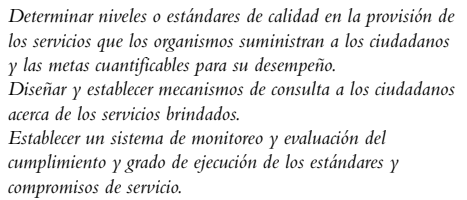 \\
\hline & $\begin{array}{l}\text { Apertura de Datos } y \\
\text { Transparencia Activa }\end{array}$ & 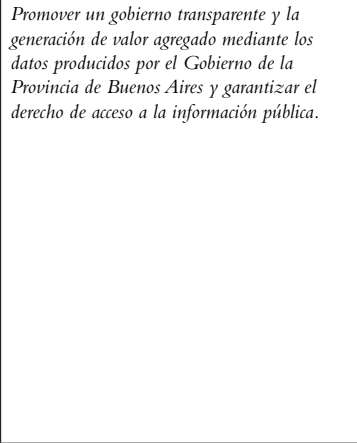 & 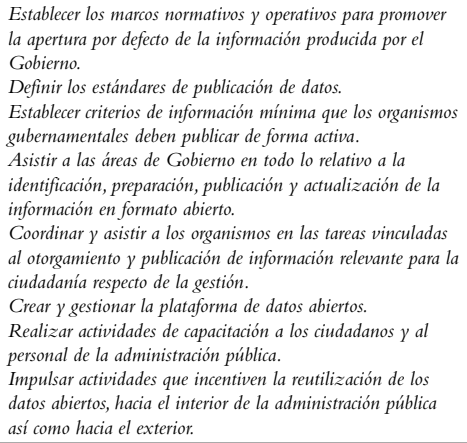 \\
\hline & $\begin{array}{l}\text { Colaboracióñ } \\
\gamma \text { PPartiopion } \\
\text { Ciuddadana }\end{array}$ & 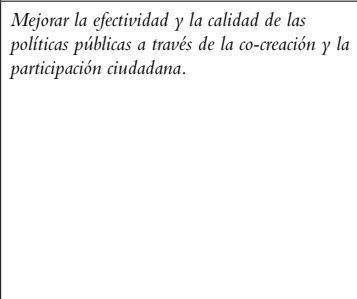 & 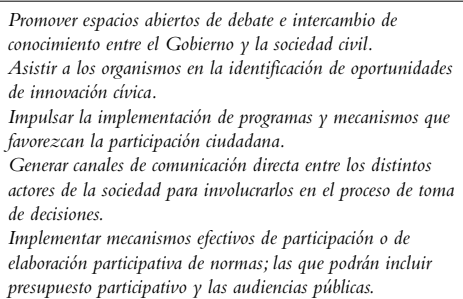 \\
\hline
\end{tabular}




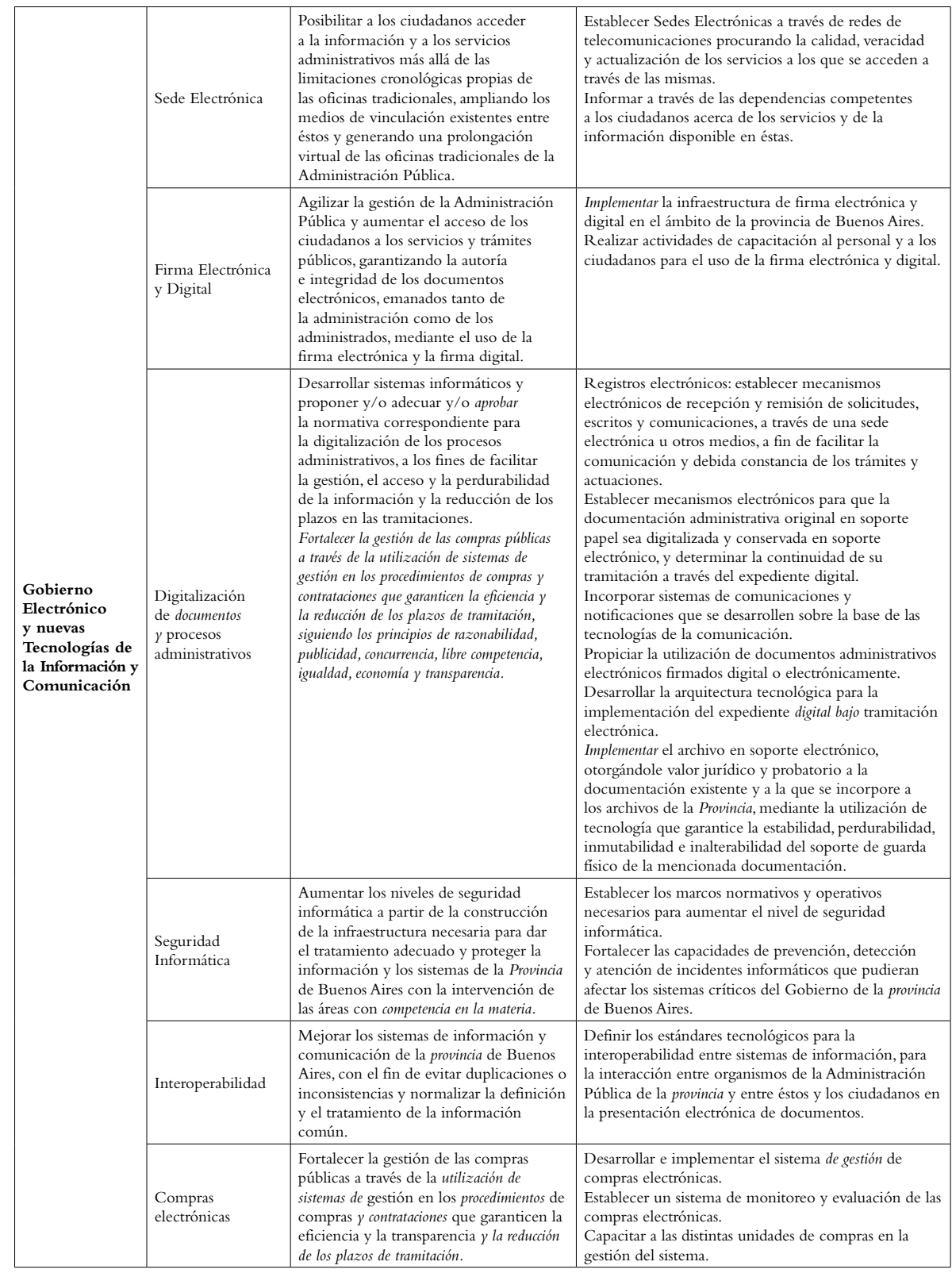

Fuente: Elaboración propia.

Nota 1: No es una cita textual sino una síntesis que elimina especialmente los procedimientos internos neNota 2: En cursiva aparecen los cos

Estratégico de Modernización respecto al texto de la Ley de la Ciudad Ley $\mathrm{N}^{\circ}$ 3304.828 que aprueba el Plan de Modernización de la Administración Pública del Gobierno de la Ciudad. Para la comparación se utilizó https://text-compare.com
Los artículos finales del Plan refieren a la posibilidad del uso de la firma electrónica o digital, cuando ya se encontraba autorizado por otra Ley provincial, a la que no refiere $\left(\text { Ley } \mathrm{N}^{\circ} 13.666\right)^{40}$.

La Ley se encuentra reglamentada por el Decreto $N^{\circ}$ 1018/16 del 22 de agosto de este año. Sus considerandos insisten con argumentos ya señalados por la Ley. En el Decreto sólo se reglamentan los aspectos vinculados a los Compromisos de Desempeño (una de las actividades del Componente "Sistema de gestión por resultados" dentro del instrumento de los Planes Operativos Anuales) y a la "Ventanilla Única de Trámites", uno de los instrumentos de gestión dentro del Componente de los mecanismos de atención ciudadana y participación.

El Anexo II y III del Decreto se vincula a la aprobación del Sistema de Gestión Documental Electrónica Buenos Aires (GDEBA) que actuará como sistema integrado de caratulación, numeración, seguimiento y registración de movimientos de todas las actuaciones y expedientes del Gobierno de la provincia de Buenos Aires. Este sistema, es similar al utilizado en el Gobierno de la Ciudad de Buenos Aires, conocido como Sistema de Administración de Documentos Electrónicos (SADE).

\section{Conclusiones}

El tiempo transcurrido desde su sanción es demasiado breve para analizar los resultados de la ley y por ello nos propusimos realizar un análisis normativo del contenido y de los antecedentes. Con estos fines, estudiamos los anteriores proyectos de modernización en la Provincia en años recientes y señalamos cómo la responsabilidad del mal funcionamiento de la Administración Pública de la Provincia se asigna a su burocratización, identificada como modelo ineficiente, lento, deshumanizado de gestionar lo público.

A pesar de que la Gobernadora, en su discurso de apertura de las sesiones ordinarias, identificó como un sistema de administración obsoleto, burocrático y lento, también advirtió las deficiencias contextuales y estructurales de la provincia. Sin embargo, el proyecto de ley enviado y aprobado por unanimidad identifica los problemas de la administración pública sólo en el propio modelo burocrático. En efecto, la Ley que aprueba el Plan Estratégico de Modernización de la Administración Pública responde a

la imperiosa necesidad de adecuar los procesos administrativos a los sistemas de calidad, herramientas informáticas y buenas prácticas de gestión administrativa.

A través de los objetivos a alcanzar y sus principios rectores, leídos como metas no alcanzadas, se infiere ese mismo diagnóstico: falta planificación, ausencia de control 
y previsión de resultados; opacidad, despilfarro, irracionalidad, ineficiencia, ineficacia, obsolescencia, corrupción, inequidad, descoordinación, etc. ${ }^{41}$

Los supuestos teóricos de la ley también dan cuenta de la continuidad de las políticas de la Nueva Gestión Pública respecto a los anteriores proyectos: planificación, compromisos de desempeño, gestión por resultados, evaluación, descentralización, flexibilidad estructural y laboral

La neutralidad de las políticas también aparece en la Ley, no sólo con los "instrumentos de gestión" sino también con los "Planes Estratégicos de Gestión" y los "Compromisos de Desempeño" y sin embargo, no hay elementos de gestión más políticos que éstos pues las pautas, metas, control y evaluación de las políticas públicas deben ser definidas por un superior. El quién aparece claro en la Ley de Modernización, el entonces Ministerio de Coordinación y Gestión Pública impulsor de la norma, que así y con la obligatoriedad de su refrenda en todos los Decretos, se constituía en verdadero jefe de la Administración Pública. ${ }^{4}$

A pesar de que la ley también habla del concepto de reforma, pretende ser un corte con lo anterior y en este sentido se plantea como un verdadero proyecto de modernización con objetivos que exceden a la mera administración, tal como se identifica el Plan. En efecto, no sólo en el texto se utilizan dos veces en forma indistinta el concepto de modernización de la Administración y modernización del Estado sino que varios de los objetivos exceden el mero saber hacer del Estado. El corte, a nuestro juicio, es tan abrupto que el principio de legalidad, tan central para el modelo burocrático, no aparece mencionado como ningún principio, criterio, ni eje de la Ley de Modernización.

En el diagnóstico de las causas que justifican la reforma nada se dice de las deficiencias propias de un modelo administrativo que no ha alcanzado los requisitos mínimos del modelo burocrático (acceso por concurso, carrera administrativa, profesionalización, salarios acordes a la responsabilidad del cargo, apego al criterio de la legalidad, capacitación permanente, relaciones impersonales, estabilidad y división del trabajo, entre otras), de la infraestructura fisica, tecnológica o dependencia presupuestaria crónica o los conflictos propios del contexto donde se pone en juego la reforma (complejidad en un vasto y heterogéneo territorio con su gran planta administrativa con casi 600.000 empleados entre permanentes, transitorios, contratados, becarios, pasantes, tercerizados, etc., con 17 cajas liquidadoras propias, 18 estatutos laborales, etc., etc.). ${ }^{43}$

La comparación legal texto a texto arroja una coincidencia exacta de 1.571 palabras entre la de la ciudad y la de la provincia, lo que supone un $39 \%$ del contenido de la Ley de la Ciudad y un 53\% de la ley de la Provincia; es decir, poco más de la mitad de la Ley bonaerense es la Ley de la ciudad de Buenos Aires. ${ }^{44}$
Las diferencias contextuales entre ambas Jurisdicciones (sólo piénsese en salario, formación, carrera y tamaño de la planta) son demasiado grandes como para dar por supuesto que el eje de la transformación sea normativo o informático. Habíamos señalado que entre los fundamentos de la Ley se afirmaba que modernizar la Provincia conllevaría desplegar servicios innovadores en el sistema de salud, seguridad, escuelas y producción pero en ningún lugar hay referencia alguna a estas temáticas sino a los aspectos propios del servicio administrativo con lo cual se confirma esta visión que pone el acento en el modelo burocrático y no en las políticas. Que la Ley no haya delineado aspectos vinculados con a la carrera administrativa o a la restructuración salarial o que la reglamentación de la Ley no haya fijado aspectos referidos a la capacitación o a la desconcentración y la descentralización territorial, que la propia ley prevé, es otra pauta.

A la fecha, lo único certero es un doble proceso: un continuum en el tiempo de los diagnósticos y soluciones de las políticas de la Nueva Gestión Pública que han atravesado, con un discurso teórico simple y efectista, distintos gobiernos provinciales; por otro lado, el mimetismo o "isomorfismo institucional" en la terminología de DiMaggio y Powell (1999) que ha implicado "tomar" el modelo de la Ciudad de Buenos Aires para "implantarlo" en la Provincia sin una exploración de las diferencias entre una y otra Jurisdicción. Si bien no es la intención de este trabajo hacer un análisis proyectivo, no podemos dejar de advertir que este tipo de políticas encuentran una fuerte resistencia organizacional y pueden fracasar, a pesar de las buenas intenciones, cuando se sustentan en errores de diagnóstico. 
${ }^{1}$ Según la investigación referenciada, el distrito de Buenos Aires está subrepresentado en aproximadamente un $9,5 \%$ en su magnitud de distrito.

${ }^{2}$ Los 70 diputados elegidos por la Provincia de Buenos Aires, ocupan poco más del 27\% del total de la Cámara baja ${ }^{3}$ Su peso específico en el contexto nacional la hace estar presente en cualquier de los grandes acontecimientos nacionales. Ha senalado Ollier (2011) una peculiar imbricacion, con referencia al caso de la Provinci de Buenos Aires, de las instancias nacional y provincial, tanto por el rol preponderante de esta Provincia en la política nacional como del impacto decisivo de la politica nacional en su territorio pero también por un natural proceso de cooperación entre ambas jurisdicciones. En efecto, su caracter des/estabilizador sobre la queda del cobierno nacional de "decidir" los candidatos a los cargos provinciales Por otro lado, se advierte en las últimas décadas la "nacionalización" de los discursos, políticas y acciones de la Provincia. Por ́́ltitime, unque no menos evidente, ambas Jurisdicciones entablan distintos procesos colaborativos que reconocen esta tensión relacional.

${ }^{4}$ Obviamente, además contribuyó la estrategia del kirchnerismo de vinculación directa con los intendentes para su construcción política. Pero sin ese proceso de desinstitucionalización, la estrategia hubiera fallado. 'En el Diccionario Pasquino (1982) entiende a la modernización como aquel conjunto de modificaciones operadas en las esferas política, económica y social que vienen caracterizando los dos últimos siglos.

'Ya estudiados por, ejemplo, en Durkheim en su tesis doctoral, La división del trabajo social.

Como agrega este autor, necesariamente el proceso de modernización va asociado al de secularización, puesto que si todo puede (o parece ser) modificado por la acción humana, lo sagrado, lo inmutable, los mitos se desvanecen.

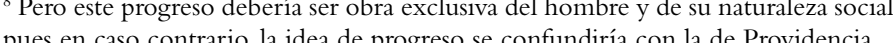

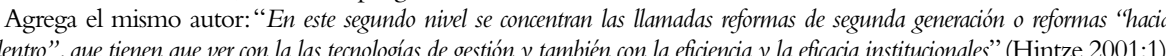
De una forma similar, Blautman ha dicho que mientras que la Reforma del Estado implicaría cambios estructurales en el ámbito social, político y económico, la Reforma Administrativa produciría modificaciones, sustancialmente en las formas de gestión del aparato público (Blutman, 1998). Echebarria Ariznabarreta (2000), por su parte, distingue las reformas institucionales, que son las que afectan el diseño y funcionamiento de las instituciones de las reformas sustanciales que afectan el contenido de la acción pública, redefiniendo su finalidad, objetivos y alcance.

Sin embargo, es Estados Unidos el principal pais promotor del modelo para la nueva gerencia pública, partir de la importante difusion alcanzada por la conocida obra Reinvención del Gobierno, de Osborne y Gaebler (1994) y yus principios de la modernización, denominados por las "Cinco R": "Reestructuración",

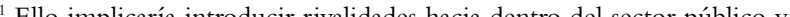

de flexibilidad laboral nunca antes vistos en las administraciones públicas latinoamericanas.

${ }^{12}$ Los modelos de reforma, los sistemas informáticos y las TIC no son políticamente neutras y pese a que se presentan sólo como herramientas, los procesos de reforma y m
últimos años también han sido conflictivos en todo Latinoamérica.

${ }^{13}$ De acuerdo a Zeller (2007) se identifica como "de primera generación" las reformas vinculadas con la transformacion estructural de los roles estatales, mientras que las destinadas a la mejora del funcionamiento de las instituciones estatales se conceptualizaron como de "segunda generación". Otra diferenciacion, ya clasica es la de Oszlak (2003) quien señala que en las reformas de primera generación -o hacia fuera - el Estado deja de hacer "algo" (v.g. producir bienes, prestar servicios, regular el comportamiento de ciertos actores), siendo reemplazado en su ejecución por un tercero (incluyendo a la "mano invisible " del mercado). El Estado prescinde totalmente

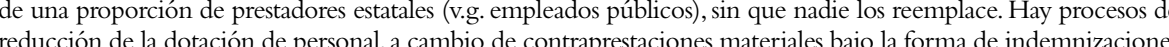
por retiro voluntario jubilación anticipada. Pero son también parte de un proceso de "reestructuración" del Estado ${ }^{14} \mathrm{La}$ Ley $\mathrm{N}^{\circ} 11.184$ fue prorrogada por el término de un año mediante la Ley $\mathrm{N}^{\circ} 11.369 \mathrm{y}$ por la Ley $\mathrm{N}^{\circ}$ 11.489 , por otro período

Se preveía la prescindibilidad de los agentes con una indemnización equivalente a la prevista en el artículo 247 de la Ley Nacional de Contrato de Trabajo -Ley N $N^{\circ} 20.744-$ y un régimen de jubilación de excepción, ${ }_{16}$ un regimen de pasividad anticipada y un sistema de retiro voluntario. Humanos; c) Proyectos para la mejora de la gestión; d) Desburocratización de la Administración Pública; y e) Nuevas Tecnologías de Gestión
${ }_{17}$ Los componentes del prog ${ }^{17}$ Los componentes del programa eran:a) Régimen de Acceso a la información Administrativa;b) Régimen de la ética
en el ejercicio de la Función Pública; c) Desburocratización de la Administración Pública Provincial y Control Social.
${ }^{18}$ Contra la elaboración de un compromiso de cumplimiento de políticas, objetivos y metas debidamente de las flexibilidades otorgadas ante su incumplimiento.

${ }^{19}$ En efecto, los bloqueos y vetos se han encontrando más bien dentro del partido gobernante por el poder de los intendentes del conurbano y de algunos legisladores del propio partido.

20 "A fines de 2004 la division en el bloque de legisladores oficialistas bonaerenses, dejando en minoría a los partidarios del Gobernador F Solá, aliado del Presidente, y la negativa a incluir en la aprobación del presupuesto provincial la delegación de poderes en el ejecutivo tal como sucedia en el ambito nacional, dieron la tónica de un conflicto creciente que preanunciaba la ruptura que se producirí para las elecciones legislativas de 2005. Luego de que esa ruptura se produjera, el oficialismo
se encontró en minoría en varias oportunidades al menos durante el segundo semestre de 2005 hasta que se redujo se enconití en minoria en'" (Cheresky 2006:48). Ello generó conflictos emestre de 2005 y hasta que se produjo lo y el Pode Le señalar que esa tensión moldeó el estilo de gobierno.

${ }^{21}$ La excepción fue la dura derrota legislativa en territorio bonaerense del Frente para la Victoria en 2009 (más por su impacto político que por los guarismos), donde asumió carácter de candidato a legislador "testimonial" junto a Néstor Kirchner. En Piana y Baeza (2013) analizamos la estrategia de marketing político de

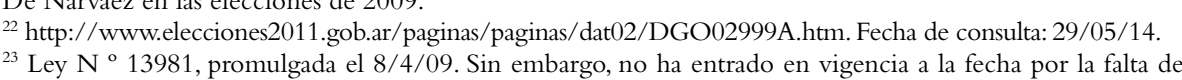
reglamentación

o-Ley $\mathrm{N}^{\circ} 7.764 / 7$

作w.hcdiputados-ba.gov.ar/proyectos/11-12PE10.do,. Fecha de consulta: 12/11/2013. Entre los fines espedescentralización de ladministración Central 2 2 Fortalecer y ampliar lano anstrum muto de desconcentración y ciatividad· 3 Institucionalizar una gestión integrada de la Región Metropolitana 4 Implantar un modelo de gestión basado en soluciones tecnológicas: 5 . Reestructurar la administración, simplificando trámites y procedimientos." ${ }^{26}$ Luego define, en esta lógica, a "Las Regiones, como unidades administrativas innovadoras, se gestan como áreas del Estado altamente ejecutivas, dinámicas y flexibles" (Buenos Aires, Plan de Regionalización ..., 2011:33).

27 (Buenos Aires, Plan de Regionalización ..., 2011:44).

${ }^{28} \mathrm{http} / / /$ provincianoticias.com.ar/2016/03/01/discurso-completo-de-la-gobernadora-maria-eugenia-vidal/ 29 El Decreto fijaba límites a la cantidad de Subsecretarías y Direcciones pero también delimitaba la cantidad de asesores así como también los pasos para la aprobación de las estructuras de los Ministerios y Secretarías entre otras pautas. Las excepciones que señaláramos estuvieron dadas exclusivamente en el primer aspecto. Fecha de Boletín Oficial: 26/05/2016.

${ }^{31}$ Fechado el 28/3/16 y publicado el 6/4/16.

32 Fechado el 30 de mayo de 2016 y publicado el 24/6/16. Antes de la aprobación de la Ley, ya entre las 政 y Gestión Pública, se la facultaba para "8. Actuar como órgano rector del/los sistema/s de soporte de gestión interno $y$ de atención al ciudadano que establezca el Gobierno Provincial con el objeto de implementar el Plan Estratégico de Modernizacion de Administraión Publica Provincial" (Decreto No 341/16 promulgado el 12/04/2016 y publicado el 29/04/2016), acción que no estaba en el diseño original de la estructura ministerial, Decreto $\mathrm{N}^{\circ}$ $39 / 15 \mathrm{~B}$, por lo que se deduce que en ese periodo se diseñó o definio esta polític

${ }_{33}^{33}$ Fecha de Sanción: 07/07/2016; Fecha de Promulgación:21/07/2016; Decreto de Promulgación: 842/2016, Fecha de Boletin Oficial: 29/07/2016.

列 de economía, eficiencia y eficacia (art. $2^{\circ}$ Anexo del Plan).

${ }^{36}$ Existe, sin embargo, un Capítulo, el II, no enumerado entre los tres componentes que es identificado como "Simplificación de la Administración Pública" que puede deberse a un error en la enumeración de los Componentes del Plan o bien una inserción errónea en el presente Título, pues no cumple con la consigna de identificar Objetivos-Instrumentos-Actividades como en los otros componentes. Nos inclinamos por la segunda porque en la ley base, la de la Ciudad de Buenos Aires, no aparece como componente.

${ }^{57}$ Como dijimos, no aparece en la Ley de Modernización de la Ciudad, el componente vinculado a la simplificación administrativ.

${ }^{38}$ El Plan menciona como actividades algunas acciones colaterales o procedimientos para llegar a las actividades. de un Presupesto Pl 
${ }^{40}$ La mención a la Ley $\mathrm{N}^{\circ} 13.666$ sólo aparece en el articulado de la Ley que aprueba el Plan pero no en el Plan en sí mismo

${ }^{41}$ El mismo criterio, expresado en sentido positivo, lo identificamos en la Ley de Emergencia Administrativa y Tecnológica, que pone la causa del mal en "años de desinversión y de administraciones imperfectas" que provocan una "actuacion estatal deficiente e ineficaz".

${ }^{12}$ Conf. articulo $5^{\circ}$ de la entonces vigente Ley de Ministerios, Ley $N^{\circ} 14.805$. A la fecha, la actual Ley provincial de Ministerios vigente, Ley 14.853, eliminó el Ministerio de Coordinación y Gestión Pública y creó nuevamente el Ministerio de Jefatura de Gabinete de Ministros, asumiendo su titular no sólo la jefatur política que tenia en el Ministerio de Gobierno sino tambien la administrativa pues retiene, al igual que el dinndo ha que co ho bjo y ́rbita del Ministerio de Jefatura de Gabinete de Ministros Ge

12 regiones sanitarias, 8 secciones electorales, 25 regiones educativas, 7 regiones turísticas, 18 depar, existen judiciales (20 creados), 13 corredores productivos además de delegaciones de la Agencia de Recaudación de Buenos Aires, Instituto Obra Médico Asistencial de la Provincia, Instituto de Previsión Social de la Provincia, de la Caja de Retiro de Policías, Registro de las Personas, Seguridad Vial, del Ministerio de Trabajo, del Ministerio de Asuntos Agrarios, del Tribunal de Cuentas en diferentes localidades de la provincia (Piana 2014). Usamos la herramienta WCopyfind.4.1.5 que se encuenta disponthle $1 \mathrm{la}$ Web para descargar y hacer

\section{Referencias}

ARELLANO GAULT, David, 2001. "Dilemas y potencialidades de los presupuestos orientados a resultados: límites del gerencialismo en la reforma presupuestal". VI Congreso Internacional del CLAD sobre la Reforma del Estado y la Administración Pública, Buenos Aires, Argentina, 5-9 noviembre de 2001.

ARELLANO GAULT, David, 2003. "Nueva Gestión Pública y diseño político-institucional. Implicaciones para un país como México". Revista IAPEM, enero-abril 2003 México.

BLUTMAN, Gustavo (1998) Aproximaciones a la Reforma del Estado: cambios en Argentina entre 1989-1992. Buenos Aires: Eudeba.

BOUZA-BREY, Luis, 1999. "El poder y los sistemas políticos" en CAMINAL BADIA, Miquel (ed.), 1999. Manual de Ciencia Política. Madrid:Tecnos.

BUENOS AIRES (2010). Plan de regionalización. Un estado inteligente para la provincia del futuro. La Plata: Ed. Plan de Regionalización de la provincia de Buenos Aires.

BUENOS AIRES (2011). Plan de Regionalización de la provincia de Buenos Aires. Un nuevo paradigma de Estado. La Plata: Ed. Plan de Regionalización de la provincia de Buenos Aires.

CAO, Horacio y Roberto ESTESO (2001), "La Reforma de las Administraciones Públicas Provinciales, balance de la década de los "90 y nueva agenda" en Administración Pública y Gestión Estatal, núm. 1. Santa Fe, Argentina: Maestría en Administración Pública / FCE / UNL.

CAO, Horacio, 2008. Administración pública argentina: nación, provincias y municipios - XIII Congreso Internacional del CLAD sobre la Reforma del Estado y de la Administración Pública, Buenos Aires, Argentina, noviembre 2008.

CENTRÁNGOLO, Oscar y JIMÉNEZ, Juan Pablo, 2004. Las relaciones entre niveles de gobierno en Argentina. Revista de la CEPAL No 84, Págs. 117-151. CEPAL.

CHERESKY, Isidoro (Comp.), 2006. La politica después de los partidos. Buenos Aires: Prometeo. 
CLEARY Eda, 2006. "El eterno dilema del control de la gestión pública en América Latina y el Caribe: Desconfianza, control y castigo versus Confianza, verificación y estímulo. Una contribución hacia la contextualización del tema". Www.top.org.ar/ecgp/ FullText/000002/2726.pdff Accesado el 19 de diciembre de 2016.

DI MAGGIO Paul y POWELL Walter (Comp.), 1999. El nuevo institucionalismo en el análisis organizacional. México: FCE.

ECHEBARRIA ARIZNABARRETA, Koldo, 2000. "Reivindicación de la reforma administrativa: significado y modelos conceptuales". Ponencia presentada en el V Congreso Internacional del CLAD sobre la Reforma del Estado y de la Administración Pública, Santo Domingo, Rep. Dominicana, 24 - 27 Oct. 2000

FREUND, Julien, 1968. La Esencia de lo Político. Madrid: Editora Nacional.

GODIO, Julio, 2003. Argentina, luces y sombras en el primer año de transición: las mutaciones de la economía durante el gobierno de Duhalde. Buenos Aires: Biblos.

GROISMAN, Enrique, 1991. Políticas administrativas para el sistema democrático. Buenos Aires: CEAL.

HEEKS, Richard (ed.), 2001. Reinventing Government in the Information Age International Practice in IT-Enabled Public Sector Reform. London/New York: Routledge.

HINTZE, Jorge 2001. Gestión presupuestaria de estructuras: un instrumento para la gestión por resultados.VI Congreso Internacional del CLAD sobre la Reforma del Estado y la Administración Pública, Buenos Aires, Argentina, 5-9 noviembre 2001.

LOPEZ, Andrea (coord.), 2005. "Indicadores de Gestión para el Monitoreo de las Políticas de Modernización en el Sector Público: Revisión teórica y propuesta para su elaboración". Desarrollo Institucional y Modernización del Estado, $N^{\circ} 71$, Instituto Nacional de la Administración Pública, Dirección de Investigaciones, Junio.

OLLIER, María Matilde, 2011. Atrapada sin salida. Buenos Aires en la política nacional (1916-2007). Buenos Aires: UMSAN Edita.

ORLANSKY, Dora (1995): "Crisis y transformación del Estado en la Argentina (1960-1993)" en Cuadernos de Estudios Empresariales, $N^{\circ}$ 5. Madrid: Universidad Complutense de Madrid.
OSBORNE David y GAEBLER Ted, 1994 . La Reinvención del Gobierno: cómo el espiritu empresarial está transformando el Sector Público. Barcelona: Paidós.

OSZLAK, Oscar, 2000. El mito de Estado mínimo: Una década de reforma estata en Argentina. IV Congreso Internacional del CLAD sobre Reforma del Estado y de la Administración Pública, Santo Domingo, 2000.

OSZLAK Oscar, 2003. ¿Escasez de recursos o escasez de innovación?: la reforma estatal argentina en las últimas dos décadas.VIII Congreso Internacional del CLAD sobre la Reforma del Estado y de la Administración Pública, Panamá, 2003.

PASQUINO Gianfranco, 1982 "Modernización” en BOBBIO, N., MATTEUCI, N y PASQUINO, G. (Orgs.). Diccionario de politica. Madrid: Siglo XXI / pp. 1038-1049.

PIANA, Ricardo Sebastián (2012 b). El Estado. Un recorrido teórico por los temas de hoy. La Plata: EDULP

PIANA, Ricardo Sebastián (2012). La reforma de la Administración Pública en la Provincia de Buenos Aires. Su estudio en los años de la gestión 2002-2007. Tesis de doctorado. Disponible en:http://hdl.handle.net/10915/31545. Accesado el 05 de junio de 2016.

PIANA, Ricardo Sebastián y BAEZA, Noelia, 2013. "Candidatos a medida- ¿Cómo se construyó el candidato que le ganó a los Kirchner?” en Revista de la Facultad de Derecho y Ciencias Políticas de la Pontifica Universidad Bolivariana, Vol 44, No 119 (2013), Medellín.

PIANA, Ricardo Sebastián, 2014. El Plan de Regionalización de la Provincia de Buenos Aires. Una aproximación a sus presupuestos teóricos. Ponencia presentada en las VIII Jornadas de Sociología de la UNLP organizadas por el Departamento de Sociología de la Facultad de Humanidades y Ciencias de la Educación (UNLP) realizadas los días 3, 4 y 5 de diciembre 2014

PIANA, Ricardo Sebastián, 2015. ¿Regionalización de la Provincia de Buenos Aires o vuelta de la nueva gestión pública? en Revista Eletrônica do Curso de Direito da UFSM - v. 10, n.1.

RANDAZZO, Aníbal Florencio, 2004. Modernización el estado para fortalecer la democracia. El proceso de modernización del Estado de la primera provincia argentina. Buenos Aires: Prometeo.

REYNOSO, Diego, 2005. "Bicameralismo y sobre representación en Argentina en perspectiva comparada". Revista de la Sociedad Argentina de Análisis Politico Volumen 2 Número 1, pp.69-94. 